\title{
Frankincense improves memory retrieval and down- regulates the hippocampal synaptophysin mRNA during the development of the rat brain
}

\author{
Siamak Beheshti ${ }^{1 *}$ iD , Sahar Tohidloo ${ }^{1}$, Abolghasem Esmaeili $^{2}$ (iD \\ 1. Department of Plant and Animal Biology, Faculty of Biological Science and Technology, University of Isfahan, Isfahan, \\ Iran \\ 2. Department of Cell and Molecular Biology and Microbiology, Faculty of Biological Science and Technology, University of \\ Isfahan, Isfahan, Iran
}

\begin{abstract}
Introduction: Frankincense expands memory performance in different experimental models of learning. Nevertheless, the causal molecular mechanisms have not been well investigated. The expression levels of some of the synaptic proteins might probably change following the consumption of frankincense. The present study investigated the effect of maternal injection of frankincense during gestation and lactation periods on memory performance and the mRNA expression levels of syntaxin $1 \mathrm{~A}$ and synaptophysin in the hippocampus of the offspring rats.

Methods: Adult female Wistar rats weighing 180-220g received two doses (50 or $100 \mathrm{mg} / \mathrm{kg}$ ) of the aqueous extract of frankincense by gavage during gestation and lactation periods for 45 consecutive days, except three days after labor. The control group received water. Spatial memory was assessed in the male offspring rats using the Morris water maze. Quantitative PCR was used to measure mRNAs expression levels of syntaxin $1 \mathrm{~A}$ and synaptophysin.

Results: Frankincense improved spatial memory retrieval in the offspring rats. Data analysis by one-way ANOVA demonstrated that frankincense did not change the expression levels of the hippocampal syntaxin1A mRNA in the offspring rats. However, it significantly decreased the expression levels of the hippocampal synaptophysin mRNA.

Conclusion: The results indicate that consumption of frankincense during both gestation and lactation periods has a beneficial impact on spatial memory performance, which is accompanied by the down-regulation of the hippocampal synaptophysin mRNA. Nevertheless, this down-regulation did not change the improving effect of frankincense in memory.
\end{abstract}

http://dx.doi.org/10.32598/ppj.24.1.60

\section{Introduction}

Memory is a vital cognitive capability of all animals that permit them to adjust their behavior in response to new experiences. Some natural products can increase memory formation (Singh et al., 2013). Frankincense is an oleo gum resin from trees of the genus Boswellia. Iranian pregnant women

\section{Keywords:}

Frankincense;

Hippocampus;

Spatial memory;

Synaptophysin;

Syntaxin1A

\section{* Corresponding author:}

Siamak Beheshti

Email:

s.beheshti@sci.ui.ac.ir

Tel: +98 (31) 37932458

Received 17 July 2019;

Received in revised form 4

December 2019; Accepted 5

December 2019 
traditionally use frankincense for its memory augmenting properties on their fetus (Mahboubi et al., 2016). In this regard, a growing number of studies have shown the beneficial effects of frankincense in memory performance (Mahmoudi et al., 2011; Hosseini-Sharifabad et al., 2016). Some studies have shown that maternal injection of frankincense improves memory of the offspring. For example, oral injection of the aqueous extract of the Boswellia serrata (100 mg/kg/day) during gestation for three weeks increased the power of learning at the postlearning stage and short-term and long-term memory in the active avoidance task in rats (Hosseini Sharifabad et al., 2004). However, the underlying molecular changes are not well studied.

It is claimed that frankincense induces developmental changes in the brain, such as an increase in the volume of the neurons, along with the number of dendritic spines and subsequently improves memory formation. For example, the maternal injection of frankincense during gestation enlarged dendritic segments of neurons of the rat hippocampal $\mathrm{CA}_{3}$ area. The dendritic branching density was also higher in experimental rats relative to the control (Hosseini Sharifabad and Esfandiary, 2007). Also, the volume of the cellular layer of the dentate gyrus and $\mathrm{CA}_{3}$ and individual volume of their neurons augmented following maternal administration of frankincense during lactation (Hosseini Sharifabad and Esfandiari, 2012). Recently, we indicated an up-regulation in the expression levels of hippocampal calcium/calmodulin kinase II- $\alpha$ (CaMKII- $\alpha$ ) mRNA and an improving effect on memory retrieval in juvenile rats following maternal injection of frankincense during gestation and lactation periods (Beheshti et al., 2018).

CaMKII- $\alpha$ is a dendritic protein and a crucial component of the postsynaptic density of glutamatergic synapses and involved in spatial memory formation (Rongo, 2002). Evidence shows that the presynaptic release mechanisms play a role in long-term synaptic plasticity (Guo et al., 2010). Accordingly, it might be probable that some of the presynaptic proteins involved in neurotransmission might also involve in the improving effects of frankincense on memory performance. Syntaxin and synaptophysin are two presynaptic proteins involved in neurotransmission (Shin, 2014). Several members of the mammalian syntaxin family have been identified. Syntaxins 1, 2, 3 and 4 were the first group of syntaxins discovered. (Bennett et al., 1993). Syntaxin-1 has two isoforms, A and B which both are nervous system-specific proteins involved in the docking of synaptic vesicles with the presynaptic plasma membrane. Syntaxin-1A is most commonly found in neurons. It makes up approximately $1 \%$ of the total amount of brain proteins (Lang and Jahn, 2008). Synaptophysin is a synaptic vesicle membrane protein that expresses throughout the brain (Kwon and Chapman, 2011).

Here, we have evaluated the potential influence of maternal consumption of frankincense during gestation and lactation periods on the hippocampal mRNA expression levels of syntaxin1A and synaptophysin and spatial memory performance in the offspring rats.

\section{Materials and methods}

\section{Animals}

Totally 18 female and nine male adult Wistar rats weighing $180-220 \mathrm{~g}$ and their offspring were used. Rats were attained from the breeding colony of the Faculty of Biological Science and Technology, University of Isfahan. The animals were held in standard cages in a temperature $\left(24 \pm 1^{\circ} \mathrm{C}\right)$ wellordered room that was upheld on a 12:12 light cycle (light on at 07:00 am). Animals had free access to food and water in their home cage. The maintenance and care of experimental animals comply with National Institutes of Health guidelines for the humane use of laboratory animals and has been confirmed by the graduate studies committee of the Department of Plant and Animal Biology, University of Isfahan.

\section{Preparation of the aqueous extract of frankincense}

The aqueous extract of frankincense was prepared as formerly described (Beheshti and Aghaie, 2016). In brief, a proper amount of frankincense was pounded and soaked in distilled water. Twenty-four hours later, it was warm-heated on a $50^{\circ} \mathrm{C}$ water bath for $60 \mathrm{~min}$ and filtered before injection. The doses of frankincense (50 and $100 \mathrm{mg} / \mathrm{kg}$ ) were selected consistent with previous reports designating its effectiveness on the improvement of memory (Beheshti et al., 2018; Mahmoudi et al., 2011; Yassin et al., 2013; Beheshti and Aghaie, 2016; Beheshti and Karimi, 2016). 


\section{Behavioral experiments}

Two female rats and one male rat were retained in distinct cages to mate. After the observation of the vaginal plaques (zero-day), male rats were removed from the cage and the female rats were treated with frankincense (50 or $100 \mathrm{mg} / \mathrm{kg}$ by gavage) or tap water $(1 \mathrm{ml} / \mathrm{kg}$ by gavage) for about 20 consecutive days. Three days after labor, the mother rats received frankincense (50 or $100 \mathrm{mg} / \mathrm{kg}$ by gavage) or tap water again for another 25 consecutive days. Each group consisted of 6 juvenile rats, of which one rat was chosen randomly from each mother. Thirty days after labor, in three groups, memory performance was evaluated using a Morris water maze. In other groups, the juvenile rats were decapitated and their hippocampi were detached and frozen directly in liquid nitrogen and stored in a $-70^{\circ} \mathrm{C}$ freezer.

\section{Morris water maze procedure}

The Morris water maze task was done as previously described (Beheshti et al., 2018). In brief, twenty-four hours before the start of training, the rats were let to execute a 60s swim without the platform to be adapted to the pool. A single training session was used, which consisted of eight trials with four different starting positions that were equally distributed around the perimeter of the maze. Each rat was placed in the water facing the wall of the tank at one of the four selected starting points (north, east, south and west) and allowed to swim and find the hidden platform located in the SW quadrant (target quadrant) of the maze. Each of four starting positions was used twice in eight training sessions.

During each trial, each rat was given 60 s to find the hidden platform. After finding the platform, the animals were permitted to remain there for $20 \mathrm{~s}$ and then placed in a holding cage for 30s until the start of the next trial. After completion of training, the animals returned to their home cages. Twenty-four hour later, a retention test (probe trial) was performed. In the probe trial, the hidden platform was removed and the animal was released from the north position and allowed to swim freely for $60 \mathrm{~s}$. All of the experiments were directed between 9:00 and 15:00.

\section{RNA extraction and complementary DNA (cDNA) synthesis}

The mRNA expression inspect was executed as formerly described with some changes (Beheshti et al., 2017). In brief, the frozen right hippocampi were powdered entirely and mixed with $200 \mu$ ice-cold phosphate-buffered saline (in mmol/l: $137 \mathrm{NaCl}, 2.7$ $\mathrm{KCl}, 4.3 \mathrm{Na} 2 \mathrm{HPO} 4.7 \mathrm{H} 2 \mathrm{O}$ and $1.4 \mathrm{KH} 2 \mathrm{PO} 4)$ vortexed for $30 \mathrm{~s}$ and then distributed into aliquots. Total cellular RNA was extracted using RNX-PLUS reagent (SinaClon, Iran). The extracted RNA was treated with $1 U$ RNase-free DNase I. The integrity of the RNA samples was determined using agarose gel electrophoresis. The concentration and purity of the RNAs were determined by a Nanodrop spectrophotometer. The mean absorbance ratio at $260 / 280 \mathrm{~nm}$ was $1.78 \pm 0.02$ and at $260 / 230 \mathrm{~nm}$ was 1.8 \pm 0.02. The reverse transcription reaction was accomplished with a cDNA synthesis kit (Takara, Japan) using Oligo-dT primer, MULV reverse transcriptase and 500ng total RNA as a template consistent with the manufacturer's instructions.

\section{Quantitative PCR}

Syntaxin1A and synaptophysin were selected as target genes, and GAPDH was used as a housekeeping gene. All primers were designed using the NCBI primer design tool (Table 1). The specificity of the primers for their target sequences was checked on the NCBI website (www.ncbi.nlm.nih.gov/blast). The SYBR Green I real-time PCR assay was carried out in a final reaction volume of $10 \mu \mathrm{l}$ with $5 \mu \mathrm{l}$ SYBR Green I Master mix (Takara, Japan), 100 nmol// forward and reverse primers and 10ng cDNA. Thermal cycling was executed on the Bio-Rad (BioRad, USA) using the following cycling conditions: 30s at $95^{\circ} \mathrm{C}$ as the first denaturation step followed by 40

Table 1: The sequences of the specific primers and the respective amplicon sizes

\begin{tabular}{llll}
\hline Target & Forward primer $\mathbf{5}^{\prime} \rightarrow \mathbf{3}^{\prime}$ & Reverse primer $\mathbf{5}^{\prime} \rightarrow \mathbf{3}^{\prime}$ & Amplicon (bp) \\
\hline Syntaxin1A & CGCCACTCAGTCAGACTACC & GTGCCTGGTCTCGATCTCAC & 193 \\
\hline Synaptophysin & AGGGCCTATGATGGACTTTCTG & TCCGTGGCCATCTTCACATC & 105 \\
\hline GAPDH & CAGGGCTGCCTTCTCTTGTG & GATGGTGATGGGTTTCCCGT & 172 \\
\hline
\end{tabular}




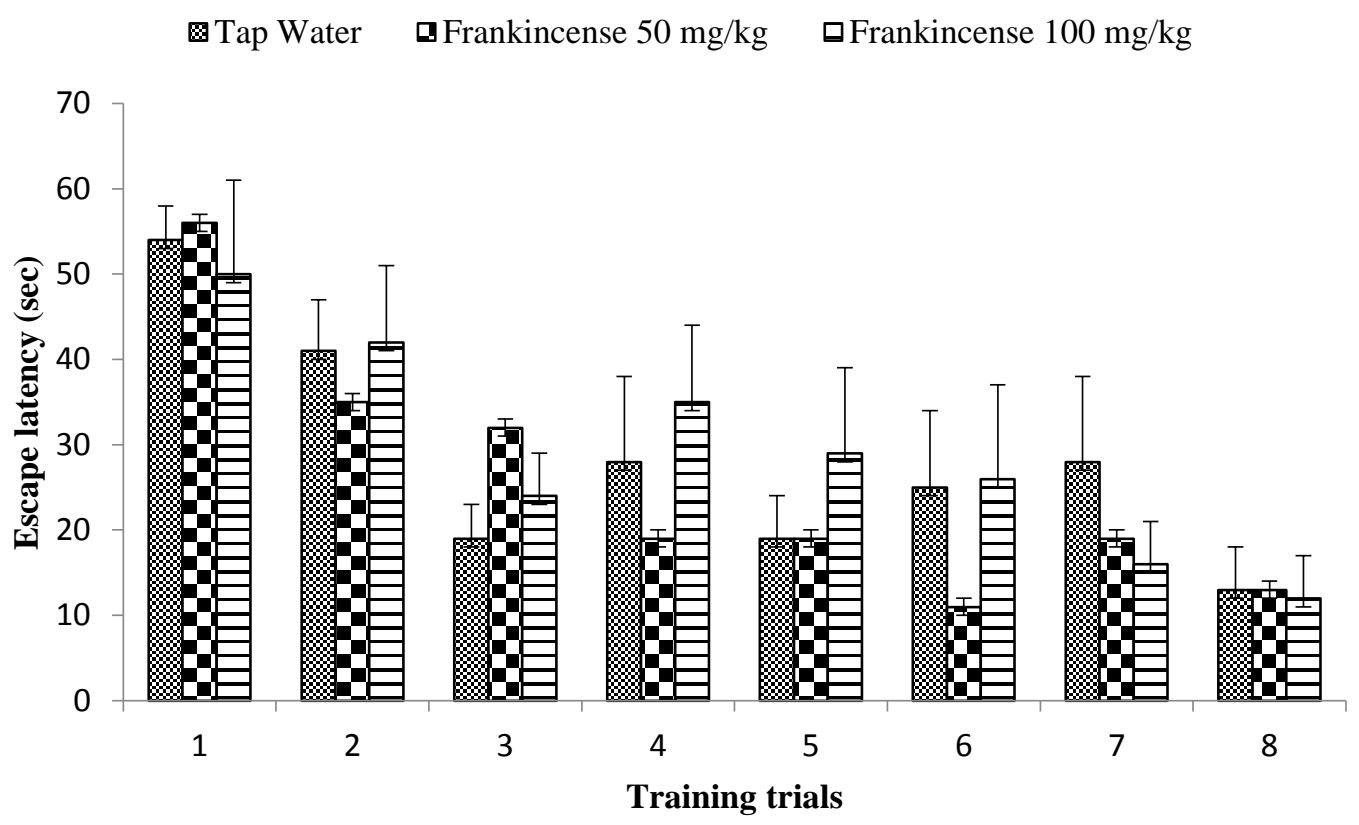

Fig.1. The effect of maternal administration of frankincense on the acquisition of memory on Morris water maze task in male offspring presenting the average escape latencies during one-day training trials. The columns represent the mean $\pm S E M$ time latencies to find the hidden platform.

cycles at $95^{\circ} \mathrm{C}$ for $5 \mathrm{~s}$ and $55.3^{\circ} \mathrm{C}$ for $30 \mathrm{~s}$. The amount of gene expression was calculated using comparative threshold cycles. The mean threshold cycle (mCT) was attained from triplicate amplifications during the exponential phase of amplification. The geometrical mean of the reference gene CT values was subtracted from the $\mathrm{MCT}$ value of the target genes to obtain $\Delta \mathrm{CT}$. The $\triangle \Delta \mathrm{CT}$ value for each sample was calculated from the corresponding $\triangle \mathrm{CT}$ values: $\Delta \Delta C T=\Delta C T$ (test sample)- $\Delta C T$ (control sample). The calculated $\Delta \Delta C T$ was converted to a ratio using the formula (Ratio $=2^{-\Delta \Delta C T}$ ) (Livak and Schmittgen, 2001).

\section{Statistical analysis}

The data are presented as mean \pm SEM. One-way analysis of variance (ANOVA) with Tukey-Kramer multiple comparison post-hoc test was done to analyze the data. In all experiments, $P<0.05$ was considered statistically significant.

\section{Results}

In the Morris water maze test, all rats indicated a significant decrease in escape latencies during training trials showing spatial memory acquisition (data analysis not displayed). Data analysis by oneway ANOVA showed no significant difference in the escape latencies between experimental groups during acquisition trials (Fig. 1). However, data analysis by one-way ANOVA showed a significant main effect of group on the time percent spent in the target quadrant during probe trial in rats whose mothers received frankincense during gestation and lactation periods. Post-hoc comparison indicated that frankincense $(100 \mathrm{mg} / \mathrm{kg})$, significantly increased time percent spent in the target quadrant compared to the control group (Fig. 2; $P<0.05$ ).

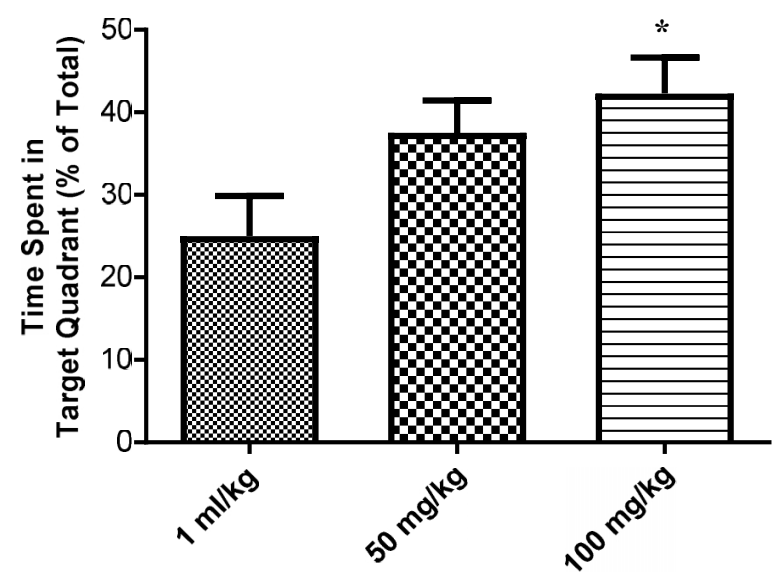

Fig.2. The effect of maternal administration of frankincense on retrieval of spatial memory in the Morris water maze task in the male offspring rats. The columns represent the mean \pm SEM time spent in the target quadrant in the 60 s probe test $\left({ }^{*} P<0.05\right.$ vs. control group). 
(A)

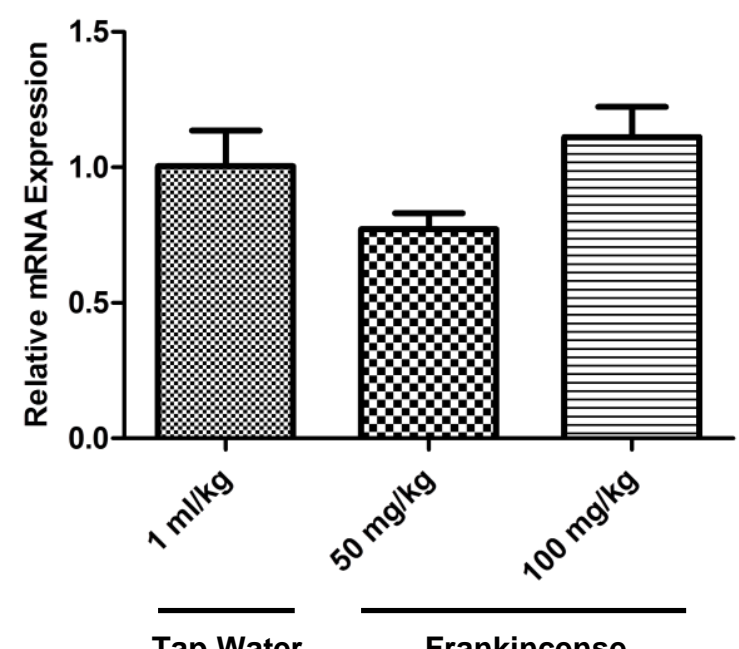

(B)

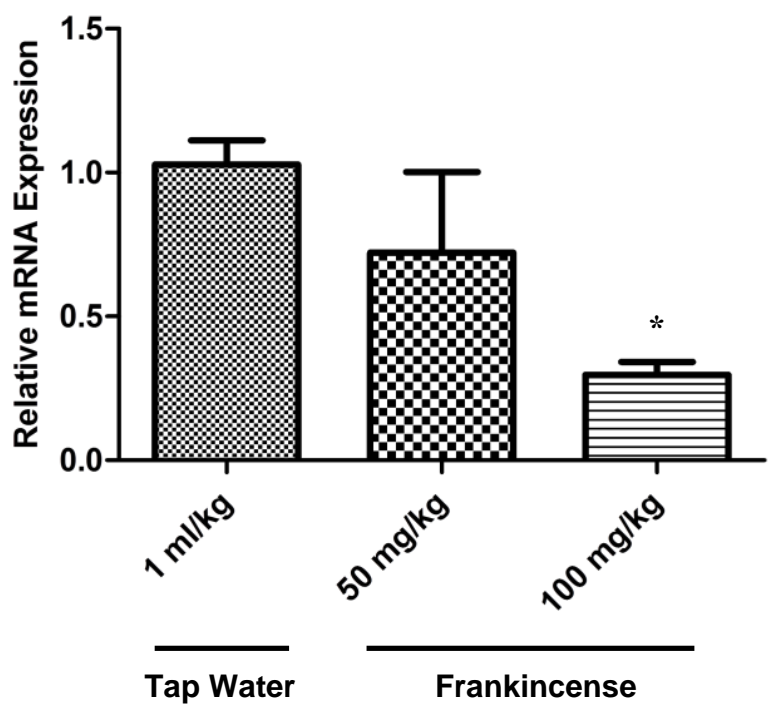

Fig.3. The effect of maternal administration of frankincense (50 or $100 \mathrm{mg} / \mathrm{kg}$ by gavage) during gestation and lactation periods at the mRNA levels of the hippocampal (A) syntaxin1A or (B) synaptophysin in the offspring rats. Syntaxin1A or synaptophysin mRNA levels were normalized to that of GAPDH mRNA. Data are expressed as mean \pm SEM $(n=5)$. Each polymerase chain reaction was done in triplicate to increase the consistency of the measurements ( $P<0.05$ vs. control group).

In quantitative PCR, melting curve analysis for the syntaxin, synaptophysin and GAPDH gene fragments showed a unique PCR product in each reaction. Mean threshold cycles of 20.97 for GAPDH, 24.22 for syntaxin and 24.16 for synaptophysin were attained. The results indicated no significant change in the expression of the hippocampal syntaxin1A mRNA levels in rats in any of the three experimental groups (Fig. 3A; $P>0.05$ ). However, it appeared a significant decrease in the expression of the hippocampal synaptophysin mRNA levels compared to the control group (Fig. 3B; $P<0.05$ ).

\section{Discussion}

The results showed that maternal administration of frankincense during gestation and lactation periods improved spatial memory retrieval in male offspring rats. The results confirm the outcomes of several earlier studies showing the potential of frankincense on the enhancement of memory. Chronic administration of a total extract of Boswellia papyrifera, caused a significant decrease in escape latency and distance traveled in the Morris water maze in rats. Also, chronic treatment with the 
aqueous extract of Boswellia serrata improved spatial learning capability in rats (Mahmoudi et al., 2011; Hosseini-Sharifabad et al., 2016). To clarify a potential molecular target in the observed results, we measured the gene expression of two putative synaptic proteins involved in the synaptic function named syntaxin1A and synaptophysin. The results showed a significant decrease in the expression of the hippocampal synaptophysin mRNA levels compared to the control group.

Synaptophysin is an integral membrane protein of synaptic vesicles. The analysis of synaptophysin interactions with other synaptic vesicle proteins and presynaptic molecules had not shown a clear function. A potential role for synaptophysin in regulating synaptic vesicle cycling had been proposed by the findings that antibodies to synaptophysin diminished neurotransmitter release in neuromuscular synapses (Alder et al., 1992). However, mice carrying a targeted deletion of the synaptophysin gene did not display any obvious phenotype to support these proposals. The anatomical construction and protein composition of the brain looked normal, and the properties of baseline synaptic transmission and short- and longterm synaptic plasticity also were unchanged compared with wild-type mice. These results had suggested that synaptophysin function is either redundant or compensated for by other proteins. It was also proposed that synaptophysin might play a delicate, needless controlling role in some aspects of synapse function that is not apparent when comparing the differences between wild-type and mutant animals (Eshkind and Leube, 1995).

On the contrary, recent studies have proposed synaptophysin as a marker of synaptic plasticity (Liu et al., 2013). Its up-regulation correlated with longterm potentiation, suggesting that the regulation of synaptophysin expression may contribute to the mechanisms underlying learning and memory (Lynch et al., 1994; Mullany and Lynch, 1997). Synaptophysin knock-out mice showed impairments in learning and memory, remarkably reduced object novelty recognition and reduced spatial learning (Schmitt et al., 2009). Another study showed that the hippocampal synaptophysin expression in aged (2224 month-old) rats was significantly lower than that in young ( 1 month old) and adult (4 months old) rats. However, its expression levels was significantly greater in the cortex of aged rats than in that of young or adult rats, and levels were similar between the three age groups in the cerebellum. Synaptophysin expression in the hippocampus was correlated with memory ability but had no relation to learning ability. In addition, synaptophysin expression in the cortex and cerebellum was not found to be correlated with learning and memory abilities (Liu et al., 2013).

There are not any in vitro or in vivo studies in the literature assessing the effect of frankincense on the expression levels of syntaxins or synaptophysin. However, based on the results of the present study, we can infer that down-regulation of synaptophysin expression levels in the hippocampus of the offspring rats, followed by maternal administration of frankincense during gestation and lactation periods did not affect its memory improving impact. The exact effect of this change needs to be clarified in future studies.

We did not assess the protein levels of synaptophysin in the hippocampus. However, studies have shown that the changes in the expression of the mRNA and protein levels of synaptophysin might not essentially correlate. For example, synaptophysin mRNA expression decreased in schizophrenia patients in some cortical areas, whereas the related protein did not change and vice-versa. The mRNA levels were decreased in the temporal cortex, but its protein did not change. Meanwhile, its mRNA did not change in the prefrontal cortex, while its protein levels decreased (Eastwood et al., 2000; Glantz et al., 2000). Therefore, the down-regulation of the synaptophysin mRNA might not necessarily result in decreased expression of its protein, which needs further experiments. Meanwhile, inferring the significance of a decreased mRNA when the level of the encoded protein is possibly preserved is not forthright. It might be owing to an altered balance of transcriptional versus translational gene regulation. For example, only a small portion of an mRNA is translationally active and being used for protein synthesis.

Frankincense has various components. The main chemical components isolated from frankincense are pentacyclic triterpenoids, tetracyclic triterpenoids, macrocyclic diterpenoids and a variety of essential oils. Pentacyclic triterpenoids are the most studied and characteristic constituents in frankincense and the main components responsible for its 
pharmacological effects. However, based on the results of the current study, it is not evident which of the components of frankincense were responsible for memory improvement or the described changes in the expression of the hippocampal synaptophysin mRNA, which requires further examinations.

Our results indicated no significant change in the expression of the hippocampal syntaxin1A mRNA levels in rats in any of the three experimental groups. Syntaxin is an integral protein of the presynaptic membrane, which is involved in the exocytosis of the neurotransmitters. Syntaxin1A knock-out mice grew normally, were fertile and showed no difference in appearance compared with control littermate. In cultured hippocampal neurons derived from these mice, the basic synaptic transmission was normal. However, the mutant mice had impaired long-term potentiation in the hippocampal slice. Meanwhile, although knock-out mice exhibited normal spatial memory in the hidden platform test, consolidation of conditioned fear memory was impaired (Fujiwara et al., 2006). The intact expression levels of the hippocampal syntaxin1A mRNA might somehow show that the down-regulation of the synaptophysin mRNA might not have necessarily occurred due to a decrease in the synaptogenesis and the number of synapses.

\section{Conclusion}

As a conclusion, our results showed for the first time that consumption of frankincense during both gestation and lactation periods could augment memory formation in the offspring rats, which was followed by down-regulation of the hippocampal synaptophysin mRNA expression levels. Nevertheless, this down-regulation did not change the improving effect of frankincense in memory.

\section{Acknowledgments}

This work was supported by grant No. 34872/94 from the Vice-Chancellorships for Research and Technology, University of Isfahan, in support of Sahar Tohidloo for obtaining her MSc degree.

\section{Conflict of interest}

The authors declare that there is no conflict of interest. The authors alone are responsible for the content of the paper.

\section{References}

Alder J, Xie ZP, Valtorta F, Greengard P, Poo M. Antibodies to synaptophysin interfere with transmitter secretion at neuromuscular synapses. Neuron 1992; 9: 759-68. https://doi.org/10.1016/0896-6273(92)90038-F.

Beheshti S, Aghaie R. Therapeutic effect of frankincense in a rat model of Alzheimer's disease. Avicenna $J$ Phytomed 2016; 6: 468-75.

Beheshti S, Ghorbanpour Skakakomi A, Ghaedi K, Dehestani $H$. Frankincense upregulates the hippocampal calcium/calmodulin kinase ii-alpha during development of the rat brain and improves memory performance. Int J Dev Neurosci 2018; 69: 44-48. https://doi.org/10.1016/j.ijdevneu.2018.06.011.

Beheshti S, Karimi B. Frankincense improves memory retrieval in rats treated with lipopolysaccharide. J HerbMed Pharmacol 2016; 5: 12-16.

Beheshti S, Zeinali R, Esmaeili A. Rapid upregulation of the hippocampal connexins 36 and 45 mrna levels during memory consolidation. Behav Brain Res 2017; 320: 8590. https://doi.org/10.1016/j.bbr.2016.11.048.

Bennett MK, Garcia-Arraras JE, Elferink LA, Peterson K, Fleming AM, Hazuka CD, et al. The syntaxin family of vesicular transport receptors. Cell 1993; 74: 863-73. https://doi.org/10.1016/0092-8674(93)90466-4.

Eastwood SL, Cairns NJ, Harrison PJ. Synaptophysin gene expression in schizophrenia. Investigation of synaptic pathology in the cerebral cortex. Br J Psychiatry 2000; 176: 236-42. https://doi.org/10.1192/bjp.176.3.236.

Eshkind LG, Leube RE. Mice lacking synaptophysin reproduce and form typical synaptic vesicles. Cell Tissue Res 1995; 282: 423-33. https://doi.org/ 10.1007/BF00318874

Fujiwara T, Mishima T, Kofuji T, Chiba T, Tanaka K, Yamamoto A, et al. Analysis of knock-out mice to determine the role of hpc-1/syntaxin 1a in expressing synaptic plasticity. J Neurosci 2006; 26: 5767-76. https://doi.org/10.1523/jneurosci.0289-06.2006.

Glantz LA, Austin MC, Lewis DA. Normal cellular levels of synaptophysin mRNA expression in the prefrontal cortex of subjects with schizophrenia. Biol Psychiatry 2000; 48: 389-97. https://doi.org/10.1016/S00063223(00)00923-9.

Guo $\mathrm{CH}$, Senzel A, Li K, Feng ZP. De novo protein synthesis of syntaxin-1 and dynamin-1 in long-term memory formation requires CREB1 gene transcription in lymnaea stagnalis. Behav Genet 2010; 40: 680-93. https://doi.org/10.1007/s10519-010-9374-9.

Hosseini Sharifabad M, Esfandiary E. A morphometeric study on $\mathrm{CA}_{3}$ hippocampal field in young rats following maternal administration of boswellia serrata resin during gestation. Iran J Basic Med Sci 2007; 10: 176-182.

Hosseini Sharifabad M, Esfandiari E. The effects of maternal administration of boswellia gum resin (frankincense) during lactation on stereological parameters of rat hippocampus. J Isfahan Med Sch 2012; 29: 2198-2207.

Hosseini Sharifabad M, Esfandiari E, Alaei H. Effects of 
frankincense aqueous extract during gestational period on increasing power of learning and memory in adult offsprings. J Isfahan Med Sch 2004; 21: 16-20.

Hosseini-Sharifabad M, Kamali-Ardakani R, HosseiniSharifabad A. Beneficial effect of boswellia serrata gum resin on spatial learning and the dendritic tree of dentate gyrus granule cells in aged rats. Avicenna $\mathrm{J}$ Phytomed 2016; 6: 189-97.

Kwon SE, Chapman ER. Synaptophysin regulates the kinetics of synaptic vesicle endocytosis in central neurons. Neuron 2011; 70: 847-54. https://doi.org/ 10.1016/j.neuron.2011.04.001.

Lang T, Jahn R. Core proteins of the secretory machinery. Handb Exp Pharmacol 2008: 107-27. https://doi.org/ 10.1007/978-3-540-74805-2_5.

Liu Y, Liang Z, Liu J, Zou W, Li X, Wang Y, et al. Downregulation of caveolin-1 contributes to the synaptic plasticity deficit in the hippocampus of aged rats. Neural Regen Res 2013; 8: 2725-33. https://doi.org/10.3969/ j.issn.1673-5374.2013.29.004.

Livak KJ, Schmittgen TD. Analysis of relative gene expression data using real-time quantitative pcr and the 2(-delta delta $\mathrm{c}(\mathrm{t})$ ) method. Methods 2001; 25: 402-8. https://doi.org/10.1006/meth.2001.1262.

Lynch MA, Voss KL, Rodriguez J, Bliss TV. Increase in synaptic vesicle proteins accompanies long-term potentiation in the dentate gyrus. Neuroscience 1994; 60: 1-5. https://doi.org/10.1016/0306-4522(94)90197.

Mahboubi M, Taghizadeh M, Talaei SA, Takht Firozeh SM, Rashidi AA, Tamtaji OR. Combined administration of Melissa officinalis and boswellia serrata extracts in an animal model of memory. Iran J Psychiatry Behav sci 2016; 10: e681. https://doi.org/10.17795/ijpbs-681.

Mahmoudi A, Hosseini-Sharifabad A, Monsef-Esfahani HR, Yazdinejad AR, Khanavi M, Roghani A, et al. Evaluation of systemic administration of boswellia papyrifera extracts on spatial memory retention in male rats. J Nat Med 2011; 65: 519-25. https://doi.org/10.1007/s11418011-0533-y.

Mullany $P$, Lynch MA. Changes in protein synthesis and synthesis of the synaptic vesicle protein, synaptophysin, in entorhinal cortex following induction of long-term potentiation in dentate gyrus: An age-related study in the rat. Neuropharmacology 1997; 36: 973-80. https://doi.org/10.1016/s0028-3908(97)00073-7.

Rongo C. A fresh look at the role of camkii in hippocampal synaptic plasticity and memory. Bioessays 2002; 24: 223-33. https://doi.org/10.1002/bies.10057.

Schmitt U, Tanimoto N, Seeliger M, Schaeffel F, Leube RE. Detection of behavioral alterations and learning deficits in mice lacking synaptophysin. Neuroscience 2009; 162: 234-43. https://doi.org/10.1016/j.neuroscience. 2009.04.046.

Shin $\mathrm{OH}$. Exocytosis and synaptic vesicle function. Compr Physiol 2014; 4: 149-75. https://doi.org/10.1002/cphy. c130021.

Singh P, Gupta A, Verma A. Herbal memory enhancer: a review. Pharma Research 2013; 10: 96-109.

Yassin N, El-Shenawy S, Mahdy KA, Gouda N, Marrie A, Farrag $A$, et al. Effect of Boswellia serrata on Alzheimer's disease induced in rats. J Arab Soc Med Res 2013; 8: 1-11. 